Voix et Images

volxetimages

\title{
Bibliographie de Gilles Hénault
}

\section{Raymond Rouleau}

Volume 21, numéro 1 (61), automne 1995

Gilles Hénault

URI : https://id.erudit.org/iderudit/201218ar

DOI : https://doi.org/10.7202/201218ar

Aller au sommaire du numéro

Éditeur(s)

Université du Québec à Montréal

ISSN

0318-9201 (imprimé)

1705-933X (numérique)

Découvrir la revue

Citer ce document

Rouleau, R. (1995). Bibliographie de Gilles Hénault. Voix et Images, 21(1),

112-122. https://doi.org/10.7202/201218ar d'utilisation que vous pouvez consulter en ligne.

https://apropos.erudit.org/fr/usagers/politique-dutilisation/ 


\section{Bibliographie de Gilles Hénault}

\section{Raymond Rouleau, Cégep de Granby Haute-Yamaska}

\section{CEuvres 1}

\section{I.1. Volumes}

Théâtre en plein air (poésie et prose), avec six dessins de Charles Daudelin, Montréal, Cahiers de la file indienne, 1946, $41 \mathrm{p}$.

Totems, avec quatre illustrations d'Albert Dumouchel, Montréal, Erta, coll. "La Tête armée", 1953, [n.p.].

Voyage au pays de mémoire, avec six eaux-fortes de Marcelle Ferron, édition de luxe à tirage limité, Montréal, Erta, 1959, 36 p.

Sémaphore, suivi de Voyage au pays de mémoire, Montréal, l'Hexagone, 1962, $71 \mathrm{p}$.

Signaux pour les voyants (poèmes 1941-1962), Montréal, l'Hexagone, 1972, 211 p.; Typo, 1984, 173 p., préface de Jacques Brault.

À l'inconnue nue, avec huit dessins de Léon Bellefleur, Montréal, Parti pris, 1984, [n.p.].

Noyade: poème, avec gravures de Monique Charbonneau, tirage limité à vingt-cinq exemplaires, Montréal, Éditions de l'atelier, 1986, 60 p.

À l'écoute de l'écoumène, Montréal, l'Hexagone, 1991, 149 p.

\section{I.2. Volumes traduits}

Hénault - Seven Poems, from le Théâtre en plein air and Totems, with versions in English by Jean Beaupré and Gael Turnbull, Ontario, ronéotypé à Iroquois Falls, distribution restreinte, été 1955, [n.p.].

1. Les textes radiophoniques et satiriques, les scénarios ainsi que les articles de journaux qui touchent les activités non littéraires de Gilles Hénault ont été écartés de cette bibliographie pour des raisons d'espace. Pour une bibliographie complète des 325 articles signés par le poète, et qui sont parus au journal Le Devoir où il était directeur des pages artistiques et littéraires de mai 1959 à août 1961, et au Nouveau Journal où il était chroniqueur de politique étrangère de novembre 1961 à janvier 1962, le lecteur consultera avec profit l'excellent ouvrage de Hugues Corriveau, Gilles Hénault: lecture de Sémaphore, Montréal, Les Presses de l'Université de Montréal, 1978, p. 145-159. 
Segnali per I veggenti, traduction et présentation de Liana Nissim, Rome, Bulzoni Editore, 1985, 217 p.

Signal for Seers, A Selection of Poems by Gilles Hénault, traduction de Ray Ellenwood, Toronto, Exile Editions, 1988, 62 p.

\section{I.3. Poèmes parus dans des volumes, périodiques ou journaux}

"Il pleut ", Le Jour, 26 août 1939.

"Le 11 novembre", Le Jour, 9 septembre 1939.

"Mane Thecel Phares", Le Jour, 16 septembre 1939.

"Promesse", Le Jour, 30 septembre 1939.

"Poèmes", La Relève, $2^{\mathrm{e}}$ cahier, $5^{\mathrm{e}}$ série, mai-juin 1940, p. 47-49.

"Réflexions de Diogène ", L'Action nationale, janvier 1941, p. 31-38.

"L'invention de la roue"; La Nouvelle Relève, vol. II, $\mathrm{n}^{\circ}$ 2, octobre 1941, p. 113-115.

"Sonnet à Nelligan", La Nouvelle Relève, janvier 1942, p. 230.

"Allégories", Gants du ciel, Montréal, septembre 1943, p. 29-35.

"Noyade", Amérique française, $\mathrm{n}^{\circ}$ 17, novembre 1943, p. 35-38.

"On tourne", "Feu sur la bête angoisse" et "Un homme à la mer", Ateliers des arts graphiques, 1949.

"Voici venir le temps", Place publique, n 1, 1951, p. 29.

"Bagne ", Place publique, no 2, 1951, p. 31.

"Engueulade" et "Graffiti", dans Patrick Straram le Bison ravi, Cabiers pour un paysage à inventer, 1959.

"L'invention de la mort ", "Petite bacchanale ", "Chanson des mégots", "Bestiaire" et "Notre jeunesse" (accompagnés d'une note biographique), dans Guy Sylvestre, Anthologie de la poésie canadiennefrançaise, Montréal, Beauchemin, 1963, p. 256-264.

"Les grands sacristains" et "Mobile", Le Devoir, vol. LVI, n ${ }^{\circ} 42,8$ avril 1965.

"Chansons des mégots" et "Notre jeunesse" (accompagnés d'une note biographique), dans Alain Bosquet, Poésie du Québec, Montréal/Paris, H.M.H./Seghers, 1966-1968-1971, p. 102-106.

"Tu m'exorcises", dans Guy Sylvestre et H.Gordon Green, Un siècle de littérature canadienne/A Century of Canadian Literature, Montréal/ Toronto, H.M.H./Ryerson Press, 1967, p. 540-541.

"Le jour du jugement ", "Câblogramme " et "Bordeaux-sur-Bagne ", La Barre du jour, $\mathrm{n}^{\text {os }}$ 17-18-19-20, janvier-août 1969, 329-335.

"L'à venir ", Les Herbes rouges, no 19, avril 1974, [n.p.]. 
"Prose du dimanche", Le Jour, 4 mai 1974.

"Écrits vains", dans Philippe Haeck, Jean-Marc Piotte et Patrick Staram le Bison ravi, "Entretien avec Gilles Hénault - 30 ans après Le Refus global", Chroniques, vol. I, n 1, janvier 1975, p. 26.

"Prose du dimanche" (texte de 1973) ainsi que d'autres textes tirés de Signaux pour les voyants, avec traduction anglaise, Ellipse, $\mathrm{n}^{\circ} 18$, 1976 , p. $8-61$.

"Voici venir le temps ", "Fraternels" et "Tu m'exorcises", dans Jacques

Cotnam, Poètes du Québec 1860-1968, Montréal, Fides, 1979, p. 158162.

"Images d'un coma ", Possibles, vol. I, no 2, hiver 1977, p. 123-128.

"Dix poèmes quasi chinois ", Estuaire, no 14, décembre 1979, p. 93-108.

"Le temps court" et "Images", Estuaire, n ${ }^{\text {os }} 32-33$, été-automne 1984, p. 91-93.

"Questions pour survivre "(fragments) et "Voisinagen, Sud, 1984.

"Questions pour survivre", Possibles, vol. X, nos 3-4, printemps-été 1986, p. 19-23.

"L'Amer à boire" (fragments), Estuaire, no 50, automne 1988, p. 61-62.

"L'Amer à boire "(fragments), dans Nicole Deschamps et Jean-Yves Collette, Brise-Lames/Antemurale, Anthologie de la poésie moderne du Québec, traduction du poème par Romeo Lucchese, Rome, Bulzoni Editore, 1989, p. 195-199.

"Avec le feu, avec le vin" et "Tu m'exorcises", dans Armand Guilmette, Anthologie de la poésie québécoise, Trois-Rivières, Éditions du Zéphyr, 1989, p. $42-47$.

"Le voyageur", "Bordeaux-sur-Bagne", "Temps des aurores du temps", "Je te salue " et "Sémaphore" (accompagnés d'une note biographique), dans Laurent Mailhot et Pierre Nepveu, La Poésie québécoise des origines à nos jours, Montréal, Éditions Typo, 1990, p. 244-251.

"Tai Tchi" (extraits), Possibles, vol XV, n 3, été 1991, p. 109-111.

\subsection{Articles de périodiques ou de journaux (préface, essais et critiques littéraires)}

"La pensée de Rémy de Gourmont sur le style", Le Jour, 23 septembre 1939.

"Du style ", Le Jour, 30 septembre 1939.

"En marge d'une pièce moderne", Le Jour, 11 novembre 1939.

"Nous les intellectuels", Le Quartier latin, vol. XIII, n 14, 24 janvier 1941, p. 4 . 
"Le crépuscule de la civilisation", Le Quartier latin, vol. XXIII, $\mathrm{n}^{\circ} 19$, 28 février, 1941, p. 2.

"Cinq mystères en forme de retable", La Nouvelle Relève, vol. I, $\mathrm{n}^{\circ} 1$, septembre 1941, p. 32-35.

"Stances à l'éternel absent par Jeannine Bélanger", La Nouvelle Relève, vol. I, $\mathrm{n}^{\circ} 1$, septembre 1941, p. 54-56.

"Axes et parallaxes par François Hertel", La Nouvelle Relève, vol. I, $\mathrm{n}^{\circ} 2$, octobre 1941, p. 113-115.

"La France", Amérique française, novembre 1944, p. 3.

"Le livre ", Amérique française, décembre 1944-janvier 1945, p. 4.

"L'humour de Jules Supervielle", Gants du ciel, no 7, mars 1945, p. 43-47.

"Lieu d'asile par Georges Duhamel", Le Canada, supplément littéraire, novembre 1946.

"Alain Grandbois", Amérique française, Vol. XII, n 6, décembre 1954, p. 474.

"La poésie et la vie" dans La Poésie et nous, Collectif, Montréal, l'Hexagone, coll. "Les voix", 1958, p. 28-42.

"Jeunes ardeurs", "Fragiles passerelles" et "Poèmes d'un mandarin - Paul Morin " dans Livres et auteurs canadiens, Montréal, Jumonville, 1961, p. 32-37.

"La poésie est mot de passe", dans Guy Robert, Littérature au Québec, Montréal, Librairie Déom, 1964, p. 71-81.

"Le droit de rêver", Quoi, vol. I, no 1, janvier-février 1967, p. 53-62.

"Amical salut à Roland Giguère", La Barre du jour, décembre-mai 1968, no 11-12-13, p. 143-144.

"Saint-Denys Garneau ou la vie impossible", Études françaises, vol. V, $\mathrm{n}^{\circ} 4$, novembre 1969 , p. 480-488.

"Une littérature nationale", Magazine littéraire (supplément Québec), Paris, $\mathrm{n}^{\circ} 134$, mars 1978 , p. 62-66.'

"La littérature française et l'écrivain québécois", Cabier de l'ACFAS, $\mathrm{n}^{\circ} 4$, 1980.

"La pêche à la ligne", préface de À l'orée de l'œeil de Roland Giguère, Montréal, Éditions du Noroît, 1981, 116 p.

"La modernité", Possibles, vol. VIII, no 3, printemps 1984, p. 145-148.

"De la poésie comme fonction de l'esprit ", Estuaire, nos 40-41, p. 83-86.

"Témoignages d'écrivains", Textes et contextes. Espaces imaginaires, $2^{\mathrm{e}}$ partie, Laval, Éditions Mondia, 1988, p. 326, 340, 343. 
"Les avatars d'une vie littéraire" dans André Laurendeau, un intellectuel d'ici, Actes du colloque publiés sous la direction de Lucille Beaudry et Robert Comeau, Québec, Presses de l'Université du Québec, 1990, p. 127-131.

\subsection{Volumes ou poèmes traduits par Gilles Hénault}

Sans parachute de David Fennario, Montréal, Éditions Parti pris, 1977, 240 p. Aussi, à Paris, chez Grasset, 1979, 239 p.

Pour une poétique de la Science-fiction de Darko Suvin, Montréal, Presses de l'Université du Québec, 1977, 228 p.

Devenir sociologue de Margaret A. Coulson et Carol Riddel, Ville, Éditions coopératives Albert Saint-Martin, 1981, 200 p.

"Rocaille: octobre" de Douglas Gordon Jones, Urgences, $n^{\circ}$ 16, mars 1987, p. 58-59.

\section{6. Écrits sur l'art}

"La question du primitivisme", Le Quartier,latin, vol. XXVI, $\mathrm{n}^{\mathrm{o}} 11$, 17 décembre 1943, p. III.

"Notes sur l'art", Le Canada, 17 octobre 1944.

"Borduas", Combat, $1^{\mathrm{er}}$ février 1947.

"Au sujet d'une exposition de Pierre Gauvreau", Combat, 22 novembre 1947.

"Discussions sur l'art", Combat, 13 décembre 1947.

"L'art en mouvement et le mouvement dans l'art", Vie des arts, $n^{\circ} 49$, hiver 1967-1968, p. 22-27.

"Le nouveau musée d'art contemporain", Vie des arts, no 51 , été 1968, p. 12-19.

"L'art contemporain a son musée", Culture vivante, $n^{\circ} 10$, août 1968, p. 14-19.

"Lumière dans l'art, lumière dans la vie ", Forces, n ${ }^{\circ}$ 7, printemps 1969, p. 38-49.

"Déclarations du sculpteur Roussil", Vie des arts, no 58, printemps 1970, p. 89-92.

"Le musée d'art contemporain", Vie des arts, n 63, été 1971, p. 34-39.

"Vasarely, vibration et irridiation", Vie des arts, vol. XVII, n 70 , printemps 1973, p. 34-77.

"Pierre Clerk, Topologie urbaine ", Vie des arts, vol. XVIII, nº 71, été 1973, p. 47-50. 
"Gérard Tremblay, le pouvoir d'intégration", Vie des arts, vol. XIX, n ${ }^{\circ} 76$, automne 1974, p. 64-67.

"Paradoxes sur les Arts et les sports", Vie des arts, vol. XXI, $\mathrm{n}^{\circ} 83$, été 1976, p. 22-27.

"Au début des années 50. Erta-Arts graphiques et poésie", Vie des arts, vol. XXII, $\mathrm{n}^{\circ} 90$, printemps 1978 , p. 20-23.

"Serge Tousignant. Recherches et métamorphoses", catalogue de l'exposition 5 attitudes, Musée d'art contemporain, 1981.

"Jacques Hurtubise, un magicien", Vie des arts, vol. XXVI, n ${ }^{\circ} 104$, hiver 1981-1982, p. 38-41.

"Charles Daudelin, aller-retour Montréal-Paris ", dépliant pour l'exposition Daudelin à Paris, octobre 1984.

"Marcel Marois, la Voix de la navette", Vie des arts, vol. XXVIII, $\mathrm{n}^{\circ} 114$, mars-avril-mai 1984, p. 53-55.

"Gilles Boisvert. Une nouvelle métamorphose", Vie des arts, vol. XXIX, $\mathrm{n}^{\circ} 118$, mars 1985, p. 42-45.

"La peinture de Borduas. Genèse et rupture", Vie des arts, vol. XXXIII, $n^{\circ} 130$, printemps 1988 , p. 26-29.

"Charles Daudelin. Profession sculpteur", Vie des arts, vol. XXXIV, $\mathrm{n}^{\circ} 135$, été 1989, p. 44-47.

"François Dallégret et l'objet de la ville", Vie des arts, vol. XXXV, $\mathrm{n}^{\circ} 142$, printemps 1991, p. 36-38.

\section{II. ÉTUDES}

\section{II.1. Études sur l'œuvre en général}

\section{II.1.1. Volumes ou chapitres de volumes}

BIDA, Constantin, Poésie du Québec contemporain, Montréal, Librairie Déom, coll. "Études slaves - Université d'Ottawa", n 2, 1968.

BOURASSA, André-G., Surréalisme et littérature québécoise, Montréal, L'Étincelle, 1977, p. 199-202. Aussi, Montréal, Les Herbes rouges, 1986.

CHATILLON, Pierre, "La naissancé du feu dans la jeune poésie du Québec ", Poésie canadienne-française, Montréal, Fides, coll. "Archives des lettres canadiennes n, Tome IV, 1969, p. 255-284.

DALLARD, Sylvie, Trois poètes exemplaires (1940-1979), Thèse de doctorat ès lettres, Québec, Université Laval, 1977, 292 p.

DE GRANDPRÉ, Pierre et VAN SCHENDEL, Michel, "Pionniers du surréel au Québec", Histoire de la littérature française du Québec, Montréal, Beauchemin, 1969, vol. 3, p. 211-217. 
GAGNON, Pierre, La Recherche des véritables signaux et la découverte de la femme sémaphore dans l'œeuvre de Gilles Hénault, mémoire de maîtrise ès Arts, Trois-Rivières, UQTR, 1975, 132 p.

GAUVIN, Lise et MIRON, Gaston, "Gilles Hénault ", Écrivains contemporains du Québec, Paris, Seghers, 1989, p. 279-284.

HAECK, Philippe, Naissances. De l'écriture québécoise, Montréal, VLB éditeur, 1979, p. 113-123 et 142-146.

HAMEL, Réginald, HARE, John, WYCZYNSKI, Paul, Dictionnaire pratique des auteurs québécois, Montréal, Fides, 1976, p. 354-355.

- Dictionnaire des auteurs de langue française en Amérique du Nord, Montréal, Fides, 1989, p. 689.

KUSHNER, Éva, "La poétique de Gilles Hénault ", Mélanges de civilisation canadienne-française offerts au professeur Paul Wyczynski, Ottawa, Éditions de l'Université d'Ottawa, 1977, p. 137-148.

MAILHOT, Laurent, La Littérature québécoise, Paris, Presses universitaires de France, coll. "Que sais-je?», 1975, 127 p.

MAJOR, Jean-Louis, "L'Hexagone: une aventure en poésie québécoise", Poésie canadienne-française, Montréal, Fides, coll. "Archives des lettres canadiennes", Tome IV, 1969, p. 175-203.

MARCOTTE, Gilles, Le Temps des poètes, description critique de la poésie actuelle au Canada français, Montréal, HMH, 1969, p. 85-89.

PELASSE, Cécile, La Recherche du pays de la poésie québécoise de 1945 à 1970, thèse de doctorat, Montréal, Université de Montréal, 1974, $473 \mathrm{p}$.

ROYER, Jean, Initiation à la poésie québécoise, Montréal, Leméac, 1989, p. 64-65.

VAN SCHENDEL, Michel, «Poésie québécoise 1960-1965: l'apprivoisement du vertige ou la rencontre de nouvelles traditions", Livres et Auteurs canadiens, Montréal, Jumonville, 1965, p. 13-22.

__ , "Poésie vivante, Gilles Hénault, Paul-Marie Lapointe", dans Gilles Marcotte (dir), Présence de la critique, Montréal, HMH, 1966, p. 203207.

\section{II.1.2. Articles de périodiques ou de journaux}

[ANONYME], "Spectacles et concerts. Gilles Hénault et Yves Préfontaine publient des poèmes ", La Presse, 16 juin 1960.

[ANONYME], "Deux lancements: de l'histoire à la poésie", Le Nouveau Journal, 19 avril 1962.

[ANONYME], "Hamelin-Hénault-Maillet, prix littéraires du Gouverneur général", La Presse, 17 avril 1973. 
ARCAND, Denys, "De l'indécrottable stupidité de nos critiques", Le Quartier latin, 5 décembre 1961.

BARBERIS, Robert, "A la semaine des lettres", Le Cabier (supplément du Quartier latin), Vol. II, $\mathrm{n}^{\circ} 22,31$ mars 1966, p. 10.

BAILLARGEON, Normand, "Le poète-phare - Le précurseur et initiateur de la modernité littéraire au Québec ", Le Devoir, 11 avril 1984.

BEAULIEU, François, "Le droit de rêver: Gilles Hénault", Métro-Express, 23 mars 1966.

BEAULIẸU, Victor Lévy, "Entre les lignes", Le Nouveau samedi, 19 novembre 1972.

BOSQUET, Alain, "Gilles Hénault ", La Nouvelle Revue française, septembre 1973, no 249, p. 71.

BOURASSA, André-G., "Gilles Hénault, éléments de biographie", La Barre du jour, nos 17-18-19-20, janvier-août 1969, p. 310-314.

BOURGAULT, Pierre, "Une littérature qui ne tient compte que des valeurs patriotiques n'est pas une littérature ", La Presse, 22 juin 1963.

BRAULT, Jacques, "Une poésie du risque", Culture vivante, vol. I, $\mathrm{n}^{\circ} 1$, 1966, p. 41-45.

CHAMBERLAND, Paul, "Fondation du territoire ", Parti pris, vol. IV, n ${ }^{\text {os }}$ 910-11-12, mai-août 1967, p. 11-42.

CLOUTIER, Cécile, "La jeune poésie au Canada français", Incidences, $\mathrm{n}^{\circ} 7$, janvier 1965, p. 4-11.

CORRIVEAU, Hugues, "Défense de toucher ", La Barre du jour, $\mathrm{n}^{\text {os }} 39-40-$ 41, printemps-été 1973, p. 58-84.

DAIGNEAULT, Claude, "Gilles Hénault et le symposium", Le Soleil, 26 juillet 1966.

DOSTIE, Gaétan, "La poésie québécoise - Gilles Hénault, prophète discret ", Le Jour, 9 mars 1974.

DUHAMEL, Roger, "Hénault, Filion et Ménard", La Patrie, 13 mai 1962.

ÉTHIER-BLAIS, Jean, DANSEREAU, Claude, KATTAN, Naïm, SYLVESTRE, Guy, "Nouveaux mythes et nouvelle sensibilité dans la littérature canadienne-française ", Le Devoir, 8 avril 1965.

FISETTE, Jean, "Gilles Hénault: Between simplicity and analogy", Ellipse, $\mathrm{n}^{\circ} 18,1976$, p. 62-71.

GAY, Paul, "La jeune poésie ", Le Droit, 22 juin 1968.

GODIN, Gérald, “À nous et pour nous", Le Nouveau Journal, 5 mai 1962.

HAECK, Philippe, PIOTTE, Jean-Marc et Patrick STARAM le Bison ravi, "Entretien avec Gilles Hénault - 30 ans après Le Refus Global", Chroniques, vol. I, $\mathrm{n}^{\circ} 1$, janvier 1975, p. 12-26. 
HALLE, Paul-André, " Le Cri nu dans la poésie de Gilles Hénault ", Coincidences, vol. I, $\mathrm{n}^{\circ} 2$, avril 1971, p. 46-55.

HAMELIN, Jean, "Gilles Hénault ou le langage nu de la poésie ", Le Devoir, 21 avril 1962.

—, "Hommage à nos écrivains", Le Devoir, 20 octobre 1962.

JASMIN, Claude, "Gilles Hénault : priorité aux artistes du pays!", La Presse (supplément), 5 mars 1966.

LANGEVIN, André, "Nos écrivains", Le Devoir, 21 juin 1947.

LAURIER, Marie, "Les prix du Québec, littérature - Le poète des rêves et de la laïcité ", Le Devoir, 4 et 5 décembre 1993.

LÉGER, Jean-Marc, "Où va la littérature canadienne-française? ", Le Devoir, 11 décembre 1948.

LESAGE, Germain, "Une éruption surréaliste", Revue de l'Université d'Ottawa, vol. XXXIV, $\mathrm{n}^{\circ}$ 3, juillet-septembre 1964, p. 322-338.

MAILHOT, Laurent, "La poésie de Gilles Hénault ", Voix et Images du pays, vol. VIII, Les Presses de l'Université du Québec, 1974, p. 149-161.

MARCOTTE, Gilles, "Deux nouvelles revues canadiennes-françaises", Le Devoir, 24 mars 1951

— , "Poèmes pour le plaisir", Le Devoir, 23 janvier 1954.

—_, "Les livres. Hénault et Filion, poètes d'aujourd'hui ", La Presse, 5 mai 1962.

MARTEL, Réginald, "Poésie d'ici ", La Presse, 11 novembre 1972.

MARTIN, Louis, "Et dans cent ans?", Le Magazine Maclean, vol. VII, $\mathrm{n}^{\circ} 7$, juillet 1967, p. 13-17.

MELANÇON, Robert, "Gilles Hénault enfin lu ", Le Devoir, 7 octobre 1978.

PALASCIO-MORIN, Ernest, "La vérité de la poésie exige un engagement", Photo-Journal, 6 au 12 novembre 1972.

PARADIS, Suzanne, "Quoi et La Barre du jour, deux revues, seize auteurs ", Le Soleil, 13 mai 1967.

POULIN, Gabrielle, "La poésie québécoise en 1972. Deuxième partie. Gilles Hénault à l'Hexagone", Relations, $\mathrm{n}^{\circ} 383$, juin 1973, p. 188-189.

RAYMOND, Louis-Marcel, "La littérature canadienne-française contemporaine ", Le Devoir, 26 novembre 1949.

SYLVESTRE, Guy, "Année littéraire 1946", Revue de l'Université d'Ottawa, mars 1947, p. 105.

__, "Livres en français. Poésie", University of Toronto Quarterly, juillet 1963, p. 491-500.

—, "Amour et poésie: Éros au pays de Québec", Le Devoir, 7 novembre 1964. 
TREMBLAÝ, Regis, "Gilles Hénault, poète en rupture de poésie", Le Soleil, 23 décembre 1972.

—, "Le poète québécois est suicidaire, selon Hénault ", Le Soleil, 6 octobre 1978.

VIATTE, Auguste, "Le renouveau de la littérature canadienne", Le Devoir, 27 décembre 1962.

II.2. Sur des poèmes ou recueils particuliers

II.2.1. Sur À l'écoute de l'écoumène

- BOURASSA, Lucie, Québec français, nº 86, été 1992, p. 18.

DUGAS, Benoît, "Du masculin au féminin", Spirale, mars 1992, p. 14.

CANTIN, Françoise, "À l'écoute de l'écoumène", Nuit blanche, septembre, octobre, novembre 1992, p. 15.

CLOUTIER, Guy, "En librairie", Le Soleil, 10 février 1992.

ISSENHUTH, Jean-Pierre, "Poésies", Le Devoir, 30 novembre 1991.

MARTEL, Réginald, "Un hommage très émouvant au poète Gilles Hénault ", La Presse, 18 novembre 1991.

ROY, G. Ross, "À l'écoute de l'écoumène", World Literature Today, été 1992.

TREMBLAY, Odile, "Gilles Hénault: la longue marche d'un poète ", $L e$ Devoir, 30 novembre 1991.

\section{II.2.2 Sur " L'invention de la roue »}

BONENFANT, Joseph, "L'Invention de la roue", Dictionnaire des cuvres littéraires du Québec (1940-1959), tome III, sous la direction de Maurice Lemire, Montréal, Fides, 1982, p. 521-522.

\section{II.2.3. Sur Sémaphore suivi de Voyage au pays de mémoire}

CORRIVEAU, Hugues, Gilles Hénault: lecture de Sémaphore, Montréal, Les Presses de l'Université de Montréal, 1978, 162 p.

DUHAMEL, Roger, "Sémaphore", Livres et Auteurs canadiens, Montréal, Jumonville, 1962, p. 40-41.

KUSHNER, Éva, "Sémaphore suivi de Voyage au pays de mémoire", Dictionnaire des ouures littéraires du Québec (1960-1969), tome IV, sous la direction de Maurice Lemire, Montréal, Fides, 1984, p. 806810.

LOCKQUELL, Clément, "Poème en prose de Gilles Hénault. Voyage au pays de mémoiren, Le Devoir, 24 décembre 1960. 
RICHER, Julie, "Trois poètes: Gilles Hénault, Jean-Paul Filion, Jean Ménard et deux romans qui tiennent le coup du temps", Notre temps, 12 mai 1962.

ROBERT, Guy, "Une demi-douzaine de chefs-d'œuvre S.V.P. Renaissance de l'Hexagone", Maintenant, juin 1963, p. 231.

VAN SCHENDEL, Michel, "Voyage au pays de mémoire", Liberté, vol. III, $\mathrm{n}^{\mathrm{o}} 13$, janvier-février 1961, p. 445-447.

\section{II.2.4. Sur Signaux pour les voyants}

[ANONYME], "Signaux pour les voyants", Le Livre canadien, 1973.

CHARBONNEAU, Alain, "La vitrine du livre", Le Devoir, 30 avril 1994.

HAECK, Philippe, "La circulation des lettres, Signaux pour les voyants", Hobo-Québec, janvier 1973.

KUSHNER, Éva, "Signaux pour les voyants", Livres et Auteurs québécois, Montréal, Jumonville, 1972, p. 136-140.

THÉBERGE, Jean-Yves, "Des signaux pour les voyants", Le Canada français, 10 janvier 1973.

\section{II.2.5. Sur Théâtre en plein air}

BOURÁSSA, André-G., "Sur le Théâtre en plein air", La Barre du jour, $\mathrm{n}^{\mathrm{os}}$ 17-18-19-20, janvier-août 1969, p. 315-328.

BRUNET, Berthelot, "Compte rendu du Théâtre en plein air", La Nouvelle Relève, octobre-novembre 1946, p. 457.

KUSHNER, Éva, "Théâtre en plein air ", Dictionnaire des ceuvres littéraires du Québec (1940-1959), tome III, sous la direction de Maurice Lemire, Montréal, Fides, 1982, p. 985-990.

\section{II.2.6. Sur Totems}

DALLARD, Sylvie, "Totems", Dictionnaires des ouvres littéraires du Québec (1940-1959), tome III, sous la direction de Maurice Lemire, Montréal, Fides, 1982, p. 1011-1014.

LEMOINE, Wilfrid, "Totems.de Gilles Hénault", Revue des arts et des lettres, Radio-Canada, 19 janvier 1954. 